\title{
A!
}

This is an electronic reprint of the original article.

This reprint may differ from the original in pagination and typographic detail.

Makkonen, I.; Hakala, M.; Puska, M.J.

\section{Calculation of valence electron momentum densities using the projector augmented-wave method}

Published in:

Journal of Physics and Chemistry of Solids

DOI:

10.1016/j.jpcs.2005.02.009

Published: 01/06/2005

Document Version

Peer reviewed version

Please cite the original version:

Makkonen, I., Hakala, M., \& Puska, M. J. (2005). Calculation of valence electron momentum densities using the projector augmented-wave method. Journal of Physics and Chemistry of Solids, 66(6), 1128-1135.

https://doi.org/10.1016/j.jpcs.2005.02.009

This material is protected by copyright and other intellectual property rights, and duplication or sale of all or part of any of the repository collections is not permitted, except that material may be duplicated by you for your research use or educational purposes in electronic or print form. You must obtain permission for any other use. Electronic or print copies may not be offered, whether for sale or otherwise to anyone who is not an authorised user. 


\title{
Calculation of valence electron momentum densities using the projector augmented-wave method
}

\author{
I. Makkonen $^{\text {a,*}}{ }^{\text {, M. Hakala }}{ }^{\text {b }}$, M. J. Puska ${ }^{\text {a }}$ \\ ${ }^{a}$ Laboratory of Physics, Helsinki University of Technology, P.O. Box 1100, \\ FIN-02015 HUT, Finland \\ ${ }^{\mathrm{b}}$ Division of X-Ray Physics, Department of Physical Sciences, P.O. Box 64, \\ FIN-00014 University of Helsinki, Finland
}

\begin{abstract}
We present valence electron Compton profiles calculated within the density-functional theory using the all-electron full-potential projector augmented-wave method (PAW). Our results for covalent $(\mathrm{Si})$, metallic $(\mathrm{Li}, \mathrm{Al})$ and hydrogen-bonded $\left(\left(\mathrm{H}_{2} \mathrm{O}\right)_{2}\right)$ systems agree well with experiments and computational results obtained with other band-structure and basis set schemes. The PAW basis set describes the high-momentum Fourier components of the valence wave functions accurately when compared with other basis set schemes and previous all-electron calculations.
\end{abstract}

Key words: C. ab initio calculations, C. X-ray diffraction PACS: 78.70.Ck, 71.15.Ap

\section{Introduction}

Compton scattering spectroscopy [1] is a method for obtaining direct information on electronic states in materials. From the scattering cross section one can extract the Compton profile $J\left(p_{z}\right)$, which, within the impulse approximation [2], is a one-dimensional projection of the three-dimensional electron momentum density $\rho(\mathbf{p})$. Compton scattering experiments are often analyzed with the help of electronic structure calculations, which are usually based on the framework of the band theory in the local-density approximation (LDA) of

\footnotetext{
* Corresponding author.

Email address: ima@fyslab.hut.fi (I. Makkonen).
} 
the density-functional theory [3] (DFT). A variety of different methods can be used to represent single-particle states, charge density and potential in practical calculations. The approximations made can have a large impact on the quality of the Compton profiles obtained.

The projector augmented-wave method (PAW) introduced by P. E. Blöchl [4] in 1994 is an elegant all-electron method generalizing both the pseudopotential method and the linearized augmented-plane-wave method (LAPW). The method includes a well-defined linear transformation from soft pseudo-valence (PS) wave functions used in the actual calculation to all-electron (AE) valence wave functions. This is very practical for the calculation of electron momentum densities, because the $\mathrm{AE}$ wave functions with the rapid oscillations can thus be easily constructed. One then obtains properly also the high-momentum Fourier components of the wave functions. The PAW method has already been applied to many computational problems that require knowledge of the $\mathrm{AE}$ wave functions or the full charge density near the nuclei, for example, to the calculation of magnetic hyperfine parameters [5], electric-field gradients [6], and optical properties of semiconductors [7].

Compton profiles or three-dimensional electron momentum densities have been calculated in the past with the DFT using, for example, the full-potential LAPW (FLAPW) method [8,9,10,11], localized basis sets [12,13], pseudowave functions [14], the orthogonalized plane-wave (OPW) method [15], the reconstruction of $\mathrm{AE}$ wave functions from pseudowave functions [16], the linear muffin-tin orbital method (LMTO) $[17,18,19]$, the full-potential LMTO method [20], and the Korringa-Kohn-Rostoker (KKR) band-structure scheme [21,22,23]. The Hartree-Fock method has also been applied [12,24], and the $G W$ approximation $[9,10,25]$, as well as quantum Monte Carlo (QMC) methods [26,27] which go beyond the DFT and the LDA. The PAW scheme has obvious advantages compared with many of these schemes. It is formally simple and both efficient and flexible in practice. First-row elements, transition metals and rareearth elements can be treated. It uses the full potential and charge density, and AE wave functions are available. The LMTO and KKR approaches are usually limited to the muffin-tin form of the potential, and the pseudopotential method suffers from transferability problems and from the loss of information on the true wave functions near nuclei. The localized basis sets may have convergence problems in the interstitial regions of solids, whereas the basis set of the PAW method is complete and takes into account the orthogonality of the valence states to the core states automatically without having to treat also the core electrons self-consistently. Formally the closest resemblance is to the OPW method, which also contains a linear transformation between PS and AE wave functions. Also the reconstruction of $\mathrm{AE}$ wave functions from PS wave functions has some similarities, e.g. the existence of soft PS wave functions and the frozen-core approximation used. However, the PAW transformation is more effective than the OPW transformation, and the reconstruction proce- 
dure of AE wave functions from PS wave functions is a more complicated and artificial way to obtain the AE wave functions than the built-in transformation of the PAW method.

In this work we focus on the PAW method as a computational tool and demonstrate its applicability to the calculation of the valence electron momentum densities by calculating Compton profiles for different systems. According to our knowledge, this is the first time the PAW method is applied to this problem. Ishibashi [28] has recently used the PAW method to calculate momentum densities of annihilating electron-positron pairs in bulk materials, and Rummukainen et al. [29] those in the case of defects is solids. We show examples of semiconducting, metallic and molecular systems, namely bulk $\mathrm{Si}$, bulk Li, bulk $\mathrm{Al}$, and the water dimer $\left(\mathrm{H}_{2} \mathrm{O}\right)_{2}$, and compare our results with experiment and other computational results. We restrict ourselves only to the non-spinpolarized case, but stress that the PAW method can also be applied to the calculation of spin-dependent momentum densities, i.e. magnetic Compton profiles $[30,31,32]$, within the impulse approximation.

\section{Method of calculation}

\subsection{Projector augmented-wave functions}

The PAW method is based on a linear transformation $\mathcal{T}$ between soft PS valence wave functions $|\tilde{\Psi}\rangle$ and corresponding $\mathrm{AE}$ valence wave functions $|\Psi\rangle$,

$$
|\Psi\rangle=\mathcal{T}|\tilde{\Psi}\rangle
$$

The transformation differs from unity by local atom-centered contributions $\hat{\mathcal{T}}_{R}$ such that

$$
\mathcal{T}=1+\sum_{R} \hat{\mathcal{T}}_{R}
$$

Each local contribution represents the difference between the AE wave function and the PS wave function and acts within some augmentation region $\Omega_{R}$ enclosing one atom. The AE wave function $|\Psi\rangle$ is expanded locally within every $\Omega_{R}$ into solutions $\left|\phi_{i}\right\rangle$ of the Schrödinger equation for the corresponding isolated atom. The index $i$ refers to the site index $R$, the angular momentum indices $(l, m)$ and an additional index $k$ referring to the reference energy $\epsilon_{k l}$. Also the PS wave function $|\tilde{\Psi}\rangle$ is expanded locally using a complete set of soft, fixed PS partial waves $\left|\tilde{\phi}_{i}\right\rangle$ and the same expansion coefficients as for the AE wave functions. There is exactly one PS partial wave $\left|\tilde{\phi}_{i}\right\rangle$ for each AE 
partial wave $\left|\phi_{i}\right\rangle$. The choice of the PS partial waves $\left|\tilde{\phi}_{i}\right\rangle$ determines the local contribution of $\hat{\mathcal{T}}_{R}$ to the transformation operator $\mathcal{T}$, i.e.,

$$
\left|\phi_{i}\right\rangle=\left(1+\hat{\mathcal{T}}_{R}\right)\left|\tilde{\phi}_{i}\right\rangle, \quad \text { within } \Omega_{R}
$$

For the transformation $\mathcal{T}$ to be linear, the expansion coefficients have to be linear functionals of the PS wave functions, i.e., inner products $\left\langle\tilde{p}_{i} \mid \tilde{\Psi}\right\rangle$ with suitable localized and fixed projector functions $\left\langle\tilde{p}_{i}\right|$. The transformation $\mathcal{T}$ can now be written as

$$
\mathcal{T}=1+\sum_{i}\left(\left|\phi_{i}\right\rangle-\left|\tilde{\phi}_{i}\right\rangle\right)\left\langle\tilde{p}_{i}\right|
$$

The final expression for the AE wave function is then

$$
|\Psi\rangle=|\tilde{\Psi}\rangle+\sum_{i}\left(\left|\phi_{i}\right\rangle-\left|\tilde{\phi}_{i}\right\rangle\right)\left\langle\tilde{p}_{i} \mid \tilde{\Psi}\right\rangle
$$

The total energy functional is modified using Eq. (5). The PS wave functions $|\tilde{\Psi}\rangle$ are the variational quantities in the PAW method, which leads to a PS Hamilton operator $\tilde{H}$ acting on the PS wave functions $|\tilde{\Psi}\rangle$. The soft PS wave functions obtained can be represented with a modest number of plane-waves, because there is no norm-conservation requirement for them and the transformation $\mathcal{T}$ leads to the modified orthogonality condition $\left\langle\tilde{\Psi}_{n}\left|\mathcal{T}^{\dagger} \mathcal{T}\right| \tilde{\Psi}_{m}\right\rangle=\delta_{n m}$. The transformation $\mathcal{T}$ incorporates the effects of the rapid oscillations of the AE wave functions within the augmentation regions $\Omega_{R}$ into every expression in the PAW method.

The AE partial waves $\left|\phi_{i}\right\rangle$ have to be orthogonalized to the core states when necessary so that the transformation $\mathcal{T}$ produces only wave functions orthogonal to the core electrons. The soft PS partial waves $\left|\tilde{\phi}_{i}\right\rangle$ must match the AE partial waves $\left|\phi_{i}\right\rangle$ outside $\Omega_{R}$. The projector functions $\left\langle\tilde{p}_{i}\right|$ are chosen so that the completeness condition $\sum_{i}\left|\tilde{\phi}_{i}\right\rangle\left\langle\tilde{p}_{i}\right|=1$ is fulfilled within every $\Omega_{R}$. This enables one to expand the PS wave function $|\tilde{\Psi}\rangle$ into PS partial waves $\left|\tilde{\phi}_{i}\right\rangle$ locally within every $\Omega_{R}$. The above condition implies that $\left\langle\tilde{p}_{i} \mid \tilde{\phi}_{j}\right\rangle=\delta_{i j}$. The construction of the partial waves and projector functions used in this work is described in detail in Ref. [33]. In practice, the partial wave expansions are truncated. Two reference energies $\epsilon_{k l}$ are used for each angular momentum quantum number $l=0,1(l=0,1,2$ for heavy alkali, alkali earth, and $\mathrm{d}$ elements).

Further details of the PAW method can be found in Refs. [4] and [34]. The implementation used in this work is based on the plane-wave code Vienna $A b$ initio Simulation Package $[35,36]$ (VASP) and its implementation of the PAW method [33]. 


\subsection{PAW valence electron momentum density}

The calculation of the momentum density of the $\mathrm{AE}$ wave functions $|\Psi\rangle$ is straightforward. The PS wave function part $|\tilde{\Psi}\rangle$ of Eq. (5) is by default represented on a regular grid in the Fourier space. Note that $|\tilde{\Psi}\rangle$ extends also to the augmentation regions $\Omega_{R}$, which simplifies the calculation in comparison to the LAPW method. The localized partial waves are functions on a radial grid multiplied by spherical harmonics $Y_{l}^{m}(\hat{\mathbf{r}})$. The calculation of their Fourier transformations is simple,

$$
\begin{aligned}
& \phi_{i=l m k R}(\mathbf{r})=\phi_{l k}(|\mathbf{r}-\mathbf{R}|) Y_{l}^{m}(\widehat{\mathbf{r}-\mathbf{R}}) \\
\stackrel{\mathcal{F}}{\Longrightarrow} \quad \phi_{i=l m k R}(\mathbf{p}) & =e^{-i \mathbf{p} \cdot \mathrm{R}} \phi_{l k}(|\mathbf{p}|) Y_{l}^{m}(\hat{\mathbf{p}}) .
\end{aligned}
$$

The radial part in the Fourier space can be written as

$$
\phi_{l k}(p)=\frac{1}{(2 \pi)^{3 / 2}} 4 \pi(-i)^{l} \int_{0}^{\infty} \mathrm{d} r r^{2} \phi_{l k}(r) j_{l}(p r),
$$

where $j_{l}(p r)$ is a spherical Bessel function. One is now able to write down the expansion of the $\mathrm{AE}$ wavefunction into plane-waves,

$$
\psi_{j \mathbf{k}}(\mathbf{r})=\left\langle\mathbf{r} \mid \Psi_{j \mathbf{k}}\right\rangle=\frac{1}{\Omega^{1 / 2}} \sum_{\mathbf{G}} C_{j \mathbf{k}}(\mathbf{G}) \exp (i(\mathbf{k}+\mathbf{G}) \cdot \mathbf{r}),
$$

where the expansion coefficient is

$$
C_{j \mathbf{k}}(\mathbf{G})=\tilde{C}_{j \mathbf{k}}(\mathbf{G})+\sum_{i}\left(\phi_{i}(\mathbf{k}+\mathbf{G})-\tilde{\phi}_{i}(\mathbf{k}+\mathbf{G})\right)\left\langle\tilde{p}_{i} \mid \tilde{\Psi}_{j \mathbf{k}}\right\rangle .
$$

Here $j$ is the band index and $\mathbf{k}$ the Bloch wave vector of the state, $\Omega$ is the volume of the supercell, G's are the reciprocal lattice points (of the superlattice), and $\tilde{C}_{j \mathbf{k}}(\mathbf{G})$ 's the plane-wave expansion coefficients of the corresponding PS wave function $\left|\tilde{\Psi}_{j \mathbf{k}}\right\rangle$. The coefficients $\left\langle\tilde{p}_{i} \mid \tilde{\Psi}_{j \mathbf{k}}\right\rangle$ in Eq. (9) can be evaluated either in the Fourier space or in the real space depending on the implementation of the code used in the computation. Because the plane-waves are eigenfunctions of the momentum, the momentum density of the state of Eq. (8) is discrete so that the momentum has only values $\mathbf{k}+\mathbf{G}$ with the probabilities determined by $\left|C_{j \mathbf{k}}(\mathbf{G})\right|^{2}$.

In the calculation of electron momentum densities it is not required to increase the kinetic energy cutoff of the PS wave functions $|\tilde{\Psi}\rangle$ from typical values of about $250-400 \mathrm{eV}$. The convergence of the $\mathrm{AE}$ wave function requires only 
the convergence of the PS wave function. The summation in Eq. (5) converges when the expansion coefficients $\left\langle\tilde{p}_{i} \mid \tilde{\Psi}\right\rangle$ have converged and the partial waves form a complete set of functions. Furthermore, it can be shown that the PAW basis set is complete whenever the plane waves form a complete set, irrespective of the partial-wave truncation [4]. The truncation, however, affects the core-valence orthogonality. It also causes errors in the expectation values and the total energy. In Eq. (9) the contribution of the PS wave function term $\tilde{C}_{j \mathbf{k}}(\mathbf{G})$ to the momentum density extends up to the momentum value $G_{\max }^{\mathrm{PS}}$ corresponding the kinetic energy cutoff of the PS wave function. The effect of the partial waves can be taken into account up to an arbitrary value $G_{\max }^{\phi-\tilde{\phi}}$.

We have tested the PAW basis set by calculating valence Compton profiles for several different isolated atoms and compared our results with the results of an AE code for atoms. We get a perfect match also at high momenta ( $p_{z}>5$ a.u.). The agreement improves systematically with increasing $G_{\max }^{\phi-\tilde{\phi}}$.

\subsection{Compton profiles}

In the Compton scattering experiment the quantity of interest is the so-called Compton profile [1]

$$
J\left(p_{z}\right)=\iint \mathrm{d} p_{x} \mathrm{~d} p_{y} \rho(\mathbf{p})
$$

where $\rho(\mathbf{p})$ is the three-dimensional ground-state momentum density given within the independent-particle model by

$$
\rho(\mathbf{p})=\frac{1}{(2 \pi)^{3}} \sum_{j}\left|\int \mathrm{d} \mathbf{r} \exp (-i \mathbf{p} \cdot \mathbf{r}) \psi_{j}(\mathbf{r})\right|^{2},
$$

where $\psi_{j}(\mathbf{r})$ 's are the occupied single-particle states. Eq. (10) is based on the impulse approximation [2]. It is assumed to be valid when the energy transferred in the scattering process is much larger than the binding energy of the electronic states involved.

In practice we calculate the momentum density of Eq. (11) by first calculating the wavefunctions using a uniform $\mathbf{k}$-point mesh dense enough to give a sufficient resolution. Then we obtain the momentum density by summing up the squares of the Fourier coefficients as

$$
\rho(\mathbf{p})=\frac{1}{\Omega} \sum_{\mathbf{k}} \sum_{j} \sum_{\mathbf{G}}\left|C_{j \mathbf{k}}(\mathbf{G})\right|^{2} \delta_{\mathbf{k}+\mathbf{G}, \mathbf{p}} .
$$


The standard way to take into account correlation effects beyond the independentparticle model in the DFT is the so-called Lam-Platzman (LP) correlation correction [37], which is isotropic within the LDA. In general, however, comparisons have shown that there are systematic anisotropic discrepancies between theory and experiment. As already noted, momentum densities have been calculated using the QMC methods [26,27] and the $G W$ approximation $[9,10,25]$. However, in the case of $\mathrm{Si}$ [26], and $\mathrm{Li}[27]$ (QMC) and $\mathrm{Li}[38,25](G W)$ the correlation correction is not found to differ appreciably from the LP corrected LDA results [31]. Wakoh et al. [39] have shown that the theoretical Compton profiles of $\mathrm{Al}$ can be fitted to the experiment using a phenomenological energy-dependent occupation number instead of the LP correction. Recently, Barbiellini and Bansil [40] have suggested a BCS-like approach in which the many-body wavefunction is constructed from singlet pair wavefunctions (geminals) by taking an antisymmetrized geminal product (AGP). The scheme is promising in being rather successful in explaining discrepancies between experiment and previous correction schemes [31]. Corrections to both the impulse approximation and to the independent particle model have been recently proposed within the Dyson orbital formalism [41].

\subsection{Computational details}

We use in our calculations the LDA based on the Ceperley-Adler [42] electron gas results as parametrized by Perdew and Zunger [43], except in the case of the $\mathrm{H}_{2} \mathrm{O}$ dimer for which a gradient-corrected functional [44] is used. We model the structures using cubic supercells. The Brillouin zone is sampled using uniform $\Gamma$-centered $\mathbf{k}$-point meshes. For the cubic solids we use the irreducible 1/48th part of the Brillouin zone. We employ the frozen-core approximation and calculate only the valence electron momentum density. The core electron momentum density is isotropic and thus does not affect anisotropy curves. However, in the case of the bulk Li also the core electrons are treated self-consistently and taken into account in the Compton profiles. We do not include the LP correction or any anisotropic correlation correction in the calculated Compton profiles. Since the LP correction is isotropic within the LDA its effect cancels out in the anisotropy curves. The Compton profiles are calculated by integrating the three-dimensional electron momentum density directly over planes in the $\mathbf{p}$-space. The theoretical profiles are convoluted with a Gaussian function with a full width at half maximum (FWHM) corresponding to experimental resolution. 


\section{Results and discussion}

\subsection{Bulk Si}

We begin by studying the anisotropy of Compton profiles of Si and compare our results with experiment and a previous computational study. Delaney et al. [16] have compared the results of their PS wave function calculations to experimental Compton profiles. Further, they have reconstructed AE valence wave functions from the PS valence wave functions and studied the effect of the reconstruction on the Compton profiles obtained. Delaney et al. used the LDA, norm-conserving pseudopotentials [45] and the reconstruction scheme by Meyer et al. [46]. They noticed that the reconstruction improves the agreement with experiment substantially. The changes in the momentum density caused by the reconstruction are not spherically-symmetric and affect the anisotropy between Compton profiles along different directions improving the agreement with the experiment. We make the same observation and get practically identical results with Delaney et al. when we calculate anisotropies using the AE and the PS wave functions of the PAW method. For the bulk Si we use the experimental lattice constant of 10.26 a.u. The $\mathbf{k}$-point mesh used is a $\Gamma$-centered $10 \times 10 \times 10$ mesh. The sampling corresponds to the momentum resolution of 0.061 a.u. The kinetic energy cutoff for the PS wave functions is $307 \mathrm{eV}$. The three-dimensional momentum density used in the calculation of the Compton profiles [Eq. (12)] covers momenta up to $p_{\max }=5.0$ a.u.

Figure 1 shows our results for the [100]-[111] anisotropy compared with the experimental result from Ref. [47]. The transformation $\mathcal{T}$ from the PS wave functions to the $\mathrm{AE}$ wave functions reduces the overestimation of the amplitudes of the peaks in the anisotropy. The [100]-[110] anisotropy is also improved but relatively less affected by the reconstruction.

\subsection{Bulk $L i$}

Bulk Li has been the subject of many high-resolution Compton scattering studies. Its Fermi surface has been studied by looking at the first and second derivatives of directional Compton profiles [48] and by reconstructing the full three-dimensional momentum density $[49,50]$. We compare our results for Li with experimental high-resolution Compton profiles and the theoretical results from Ref. [48]. The theoretical results in Ref. [48] have been calculated with the KKR band structure scheme and the LDA. The momentum density of Li has also been calculated using the LMTO method $[17,18]$, the FLAPW method $[9,10,11]$, the $G W$ approximation [9,10,25], and quantum Monte Carlo 
methods [27]. Theoretical calculations based on the LDA have failed to describe the momentum density of Li also when the Lam-Platzman correlation correction has been taken into account [48]. The discrepancies have been attributed to electronic correlation beyond the LDA $[48,49]$ or to the thermal disorder [18]. Our calculation does not properly take into account these effects. Therefore, instead of experimental data we have to use other computational results based on the LDA as benchmarks.

We calculate our results for the bulk Li using the experimental lattice constant of 6.60 a.u. The k-point mesh used is a $\Gamma$-centered $30 \times 30 \times 30$ mesh. The sampling corresponds to the momentum resolution of 0.032 a.u. The kinetic energy cutoff of the PS wave functions is $271 \mathrm{eV}$. Momenta up to $p_{\max }=$ 10.0 a.u. are taken into account when calculating the Compton profiles.

Figure 2 shows in addition to our results experimental anisotropies [48] and those calculated with the KKR [48]. The PAW and the KKR results are in perfect agreement although the muffin-tin approximation has been used in the KKR calculation. A disagreement is found between our results and the FLAPW results by Baruah et al. [11] although in principle the only major difference between the calculations is the choice of the basis set. Actually, Baruah et al. used gradient corrections to the exchange-correlation potential, but concluded that their effect on the electron momentum density is not significant. However, the FLAPW results by Kubo [9] are in good agreement with the KKR method, and thus also agree with our calculation.

\subsection{Bulk Al}

Ohata et al. [51] have measured directional high-resolution Compton profiles of $\mathrm{Al}$ and compared them with theoretical results calculated with the KKR band structure scheme within the LDA. For Al the agreement between theory and experiment was found to be better than in the case of Li. Correlation effects are less important as the electron density is higher. $\mathrm{Al}$ is also harder than $\mathrm{Li}$ and other alkali metals, which reduces the effect of thermal disorder on Compton profiles. However, because the anisotropy of Compton profiles of $\mathrm{Al}$ is very small, and the anisotropy curves and derivatives of Compton profiles contain detailed features, $\mathrm{Al}$ is the most challenging test for the PAW method presented in this paper.

For the bulk $\mathrm{Al}$ we use the experimental lattice constant of 7.65 a.u. The kpoint mesh used is a $\Gamma$-centered $60 \times 60 \times 60$ mesh. The sampling corresponds to the momentum resolution of 0.014 a.u. The kinetic energy cutoff for the PS wave functions is $296 \mathrm{eV}$. Momenta up to $p_{\max }=5.0$ a.u. are taken into account when calculating the Compton profiles. 
Figure 3 shows the calculated valence Compton profiles and their first and second derivatives compared with the experimental results of Ref. [51]. As can be expected, for the LDA, the theoretical Compton profiles are higher at low momenta $[48,16,51]$. The Lam-Platzman correlation correction would shift weight from lower to higher momenta improving the agreement with the experiment. The first and second derivatives of the Compton profiles calculated with the PAW method agree better with experiment than the KKR results by Ohata et al. (see the data in Ref. [51]). Anisotropies of the Compton profiles are studied in Fig. 4. Agreement with the KKR scheme is not as good as in the case of Li. Compared with the experiment the agreement is about as good for both theoretical methods. The PAW method does not reproduce all the features of the experimental anisotropies as clearly as the KKR scheme. However, it predicts the positions of the peaks in the anisotropies better, especially in the [110]-[100] and [111]-[100] differences.

\subsection{Water dimer $\left(\mathrm{H}_{2} \mathrm{O}\right)_{2}$}

Finally, we calculate the Compton profile of the gas-phase water dimer using the generalized-gradient approximation (GGA) by Perdew and Wang [44], and the experimental geometry by Dyke et al. [52] with the $\mathrm{O}-\mathrm{O}$ separation of $2.98 \AA$. The water dimer can be considered as a prototype system to study how the constitutive interactions of the hydrogen bond (e.g. exchange repulsion, charge transfer and polarization) are reflected in the Compton profile [12]. The gas-phase dimer represents a moderately hydrogen-bonded system, where the main oscillatory signal in the Compton profile is expected to stem from the exchange interaction of the overlapping molecular wave functions [12]. Since the binding compared to the metallic and covalent systems is much weaker, the wave functions of the two water molecules conserve to a large extent their unperturbed form. Hydrogen bonds provide thus one of the limiting cases to study the applicability of the PAW calculations to describe chemical bonding. The PAW result is calculated using a periodic supercell with a side length of 35.0 a.u. in order to minimize the interaction between the periodic images of the dimer. The k-point sampling (a $\Gamma$-centered $3 \times 3 \times 3$ mesh) used when calculating the momentum density corresponds to the momentum resolution of 0.060 a.u. In the calculation of the effective potential, however, only the $\Gamma$ point is used. The kinetic energy cutoff of the PS wave functions is $500 \mathrm{eV}$. The momentum density is taken into account up to the momentum value of $p_{\max }=13.7 \mathrm{a} . \mathrm{u}$. when calculating the Compton profile. We calculate the Compton profile of the $\mathrm{H}_{2} \mathrm{O}$ dimer also by employing localized basis functions. The same GGA functionals are used. For oxygen a triple-zeta valence plus polarization type basis set is used, and for hydrogen a primitive set augmented by one p-function in a [3s, 1p] contraction (for more details, see Ref. [13]). No experimental data is available for the directional anisotropies of the dimer. 
Figure 5 shows the anisotropy between the profile along the $\mathrm{O}-\mathrm{O}$ direction (direction of the H-bond) and that in the direction orthogonal to the mirror plane of the $\mathrm{H}_{2} \mathrm{O}$ dimer. The solid line denotes the AE PAW result, the dashed line the result calculated with localized basis functions, and the dotted line the result calculated using the PS wave functions of the PAW method. Agreement between the two AE calculations is very good, especially at high momenta. The positions of the peaks are improved discernibly when the PS wave functions are replaced by the AE wave functions. At momenta $p_{z}>2.5$ a.u. the PS wave functions fail to describe the positions of the peaks properly. At lower momenta the deficiencies in the basis sets and the residual interaction between the periodic images in the PAW method explain the slight differences in the amplitudes between the AE calculations.

\section{Summary and conclusions}

We have presented valence electron Compton profiles calculated within the DFT using the projector augmented-wave (PAW) method. The accuracy of the method has been demonstrated to be competitive with other band-structure and basis set schemes. The PAW method is ideal for the calculation of valence electron momentum densities of covalent systems. Also for systems with much weaker orbital rearrangements, such as hydrogen bonds, the PAW basis set yields reliable results. It becomes highly beneficial to use a unified approach with a well-controlled basis set and full-potential treatment for the wide variety of nonmetallic systems. The effect of the frozen-core approximation is negligible in most systems. The quality of the PAW calculations can be systematically improved by increasing the energy cutoff parameter. For metallic systems ( $\mathrm{Li}, \mathrm{Al}$ ) in the LDA, despite the coarser $\mathbf{k}$-mesh, PAW yields essentially as good results as the KKR method. Nevertheless, for metals both methods suffer from discrepancies as compared with experiment, attributed to electron correlations beyond the LDA or to thermal disorder. Our work suggests that the PAW method which is becoming ever more popular in electronic structure calculations suits well for describing a wide variety of ordered and disordered covalently or hydrogen bonded systems.

\section{Acknowledgements}

We thank Dr. Y. Sakurai for sending the experimental data on Si, Li and Al, and Prof. A. Bansil for providing the KKR Compton profiles of Li. We also thank Prof. S. Manninen, Prof. K. Hämäläinen, and Academy Prof. R. Nie-

minen for discussions. We acknowledge the generous computer resources from 
the Center of Scientific Computing, Espoo, Finland. This work has been supported by the Academy of Finland through its Centers of Excellence program (2000-2005) and contract No. 205967/201291 (MH).

\section{References}

[1] M. J. Cooper, P. E. Mijnarends, N. Shiotani, N. Sakai, A. Bansil (Eds.), X-Ray Compton Scattering, Oxford University Press, Oxford, 2004.

[2] P. Eisenberger, P. M. Platzman, Phys. Rev. A 2 (1970) 415.

[3] For a review of the density-functional theory and different computational methods, see R. M. Martin, Electronic Structure: Basic Theory and Practical methods, Cambridge University Press, Cambridge, 2004.

[4] P. E. Blöchl, Phys. Rev. B 50 (1994) 17953.

[5] P. E. Blöchl, Phys. Rev. B 62 (2000) 6158.

[6] H. M. Petrilli, P. Blöchl, P. Blaha, K. Schwarz, Phys. Rev. B 57 (1998) 14690.

[7] B. Adolph, J. Furthmüller, F. Bechstedt, Phys. Rev. B 63 (2001) 125108.

[8] C. Blaas, J. Redinger, S. Manninen, V. Honkimäki, K. Hämäläinen, P. Suortti, Phys. Rev. Lett. 75 (1995) 1984.

[9] Y. Kubo, J. Phys. Soc. Jpn. 65 (1995) 16.

[10] Y. Kubo, J. Phys. Soc. Jpn. 66 (1997) 2236.

[11] T. Baruah, R. R. Zope, A. Kshirsagar, Phys. Rev. B 60 (1999) 10770.

[12] T. K. Ghanty, V. N. Staroverov, P. R. Koren, E. R. Davidson, J. Am. Chem. Soc. 122 (2000) 1210.

[13] M. Hakala, S. Huotari, K. Hämäläinen, S. Manninen, Ph. Wernet, A. Nilsson, L. G. M. Pettersson, Phys. Rev. B 70 (2004) 125413.

[14] E. D. Isaacs, A. Shukla, P. M. Platzman, D. R. Hamann, B. Barbiellini, C. A. Tulk, Phys. Rev. Lett 82 (1999) 600.

[15] J. Felsteiner, M. Heilper, I. Gertner, A. C. Tanner, R. Opher, Phys. Rev. B 23 (1981) 5156.

[16] P. Delaney, B. Králik, S. G. Louie, Phys. Rev. B 58 (1998) 4320.

[17] A. A. Manuel, L. Oberli, A. K. Singh, T. Jarlborg, M. Peter, P. E. Mijnarends, L. P. L. M. Rabou, T. Hyodo, A. T. Stewart, J. Phys. Condens. Matter 5 (1993) 8703.

[18] S. B. Dugdale, T. Jarlborg, Solid State Commun. 105 (1998) 283. 
[19] B. Barbiellini, S. B. Dugdale, T. Jarlborg, Comput. Mater. Sci. 28 (2003) 287.

[20] C. Metz, Th. Tschentscher, P. Suortti, A. S. Kheifets, D. R. Lun, T. Sattler, J. R. Schneider, F. Bell, Phys. Rev. B 59 (1999) 10512.

[21] A. Bansil, S. Kaprzyk, Phys. Rev. B 43 (1991) 10335.

[22] S. Kaprzyk, A. Bansil, Phys. Rev. B 42 (1990) 7358.

[23] A. Bansil, S. Kaprzyk, J. Tobola, Mater. Res. Soc. Symp. Proc. 253 (1992) 505.

[24] A well-known Hartree-Fock momentum density implementation for clusters and solids is available in the CRYSTAL03 package (see http://www.crystal.unito.it).

[25] A. G. Eguiluz, W. Ku, J. M. Sullivan, J. Phys. Chem. Solids 61 (2000) 383.

[26] B. Králik, P. Delaney, S. G. Louie, Phys. Rev. Lett. 80 (1998) 4253.

[27] C. Filippi, D. M. Ceperley, Phys. Rev. B 59 (1999) 7907.

[28] S. Ishibashi, Mater. Sci. Forum 445-446 (2004) 401.

[29] M. Rummukainen, I. Makkonen, V. Ranki, M. J. Puska, K. Saarinen, H.-J. L. Gossmann, to be published.

[30] P. M. Platzman, N. Tzoar, Phys. Rev. B 2 (1970) 3556.

[31] W. Schülke, in Ref. [1], p. 22.

[32] N. Sakai, in Ref. [1], p. 289.

[33] G. Kresse, D. Joubert, Phys. Rev. B 59 (1999) 1758.

[34] P. E. Blöchl, C. J. Först, J. Schimpl, Bull. Mater. Sci. 26 (2003) 33.

[35] G. Kresse, J. Furthmüller, Comput. Mat. Sci. 6 (1996) 15.

[36] G. Kresse, J. Furthmüller, Phys. Rev. B 54 (1996) 11169.

[37] L. Lam, P. M. Platzman, Phys. Rev. B 9 (1974) 5122.

[38] W. Schülke, J. Phys. Soc. Jpn. 68 (1999) 2470.

[39] S. Wakoh, M. Matsumoto, M. Tokii, J. Phys.: Condens. Matter 12 (2000) 9725.

[40] B. Barbiellini, A. Bansil, J. Phys. Chem. Solids 62 (2001) 2181.

[41] I. G. Kaplan, B. Barbiellini, A. Bansil, Phys. Rev. B 68 (2003) 235104.

[42] D. M. Ceperley, B. J. Adler, Phys. Rev. Lett. 45 (1980) 566.

[43] J. P. Perdew, A. Zunger, Phys. Rev. B 23 (1981) 5048.

[44] J. P. Perdew, in: P. Ziesche, H. Eschring (Eds.), Electronic Structure of Solids '91, Akademie Verlag, Berlin, 1991, p. 11. 
[45] D. R. Hamann, M. Schlüter, C. Chiang, Phys. Rev. Lett. 43 (1979) 1494.

[46] B. Meyer, K. Hummler, C. Elsässer, M. Fähnle, J. Phys.: Condens. Matter 7 (1995) 9201.

[47] Y. Kubo, Y. Sakurai, Y. Tanaka, T. Nakamura, H. Kawata, N. Shiotani, J. Phys. Soc. Jpn. 66 (1997) 2777.

[48] Y. Sakurai, Y. Tanaka, A. Bansil, S. Kaprzyk, A. T. Stewart, Y. Nagashima, T. Hyodo, S. Nanao, H. Kawata, N. Shiotani, Phys. Rev. Lett. 74 (1995) 2252.

[49] W. Schülke, G. Stutz, F. Wohlert, A. Kaprolat, Phys. Rev. B 54 (1996) 14381.

[50] Y. Tanaka, Y. Sakurai, A. T. Stewart, N. Shiotani, P. E. Mijnarends, S. Kaprzyk, A. Bansil, Phys. Rev. B 63 (2001) 045120.

[51] T. Ohata, M. Itou, I. Matsumoto, Y. Sakurai, H. Kawata, N. Shiotani, S. Kaprzyk, P. E. Mijnarends, A. Bansil, Phys. Rev. B 62 (2000) 16528.

[52] T. R. Dyke, K. M. Mack, J. S. Muenter, J. Chem. Phys. 66 (1977) 498. 


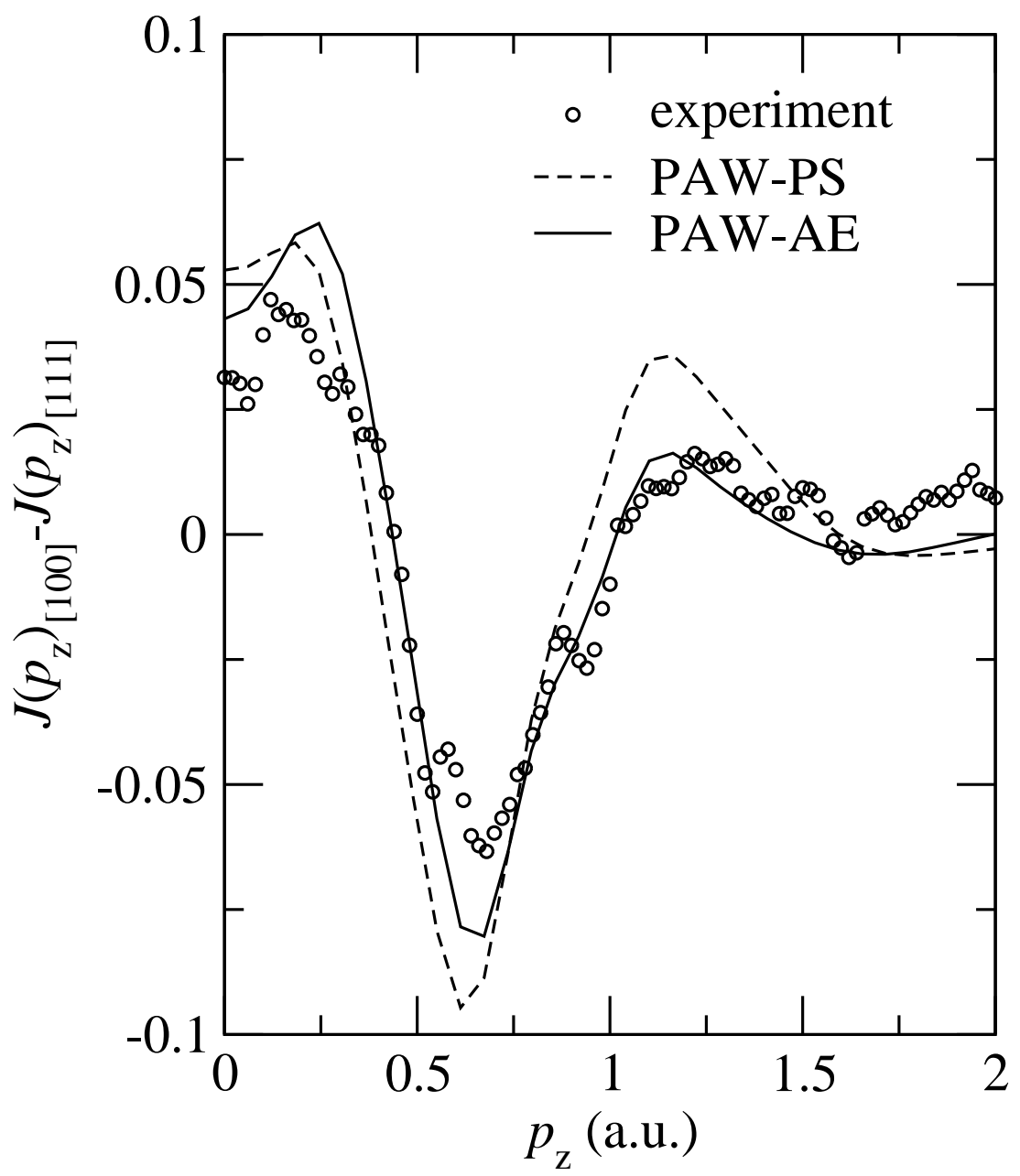

Fig. 1. Bulk Si. The anisotropy between the Compton profiles along the [100] and [111] directions. The theoretical curves are convoluted with a Gaussian function with a FWHM of 0.13 a.u. corresponding to the experimental resolution. The experimental data is from Ref. [47]. 


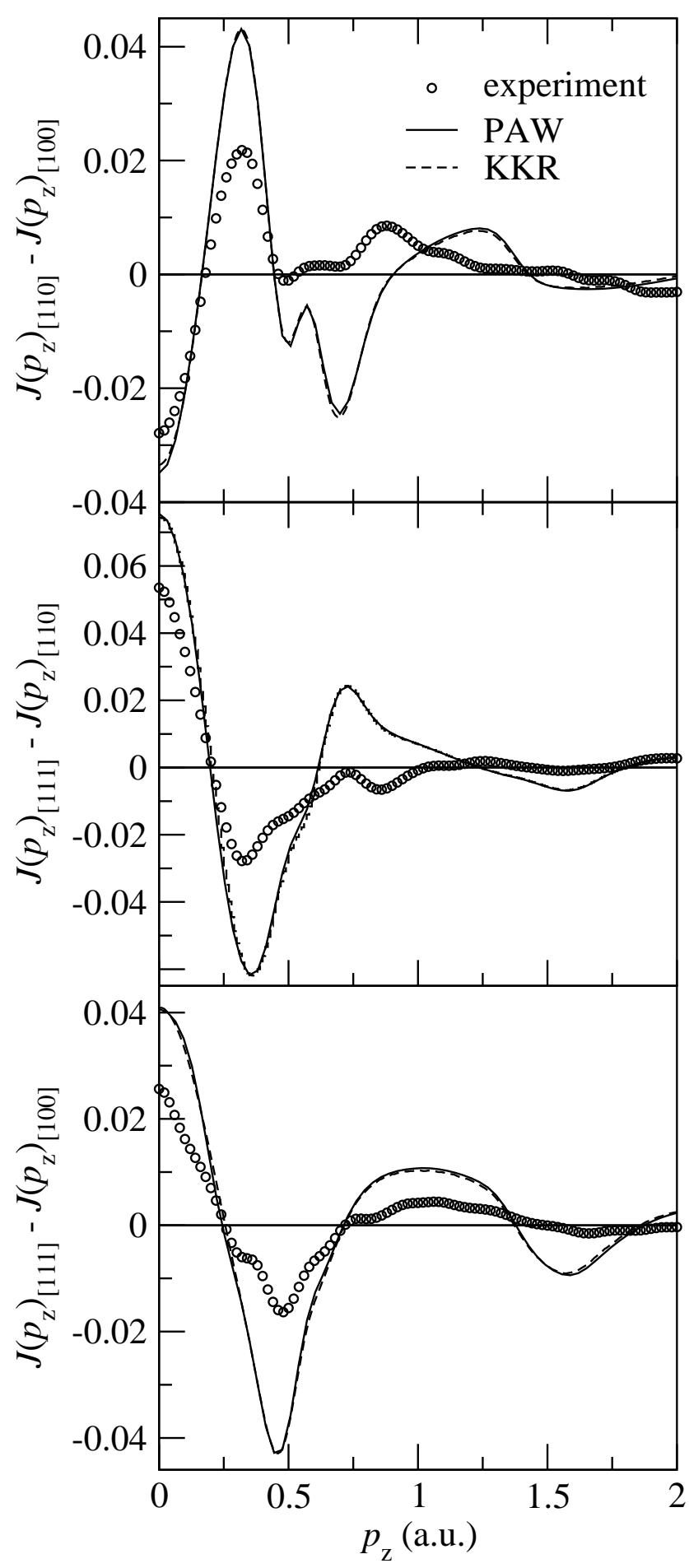

Fig. 2. Bulk Li. Anisotropies between Compton profiles along different directions. The theoretical curves are convoluted with a Gaussian function with a FWHM of 0.12 a.u. corresponding to the experimental resolution. The experimental data and the KKR result are from Ref. [48]. 


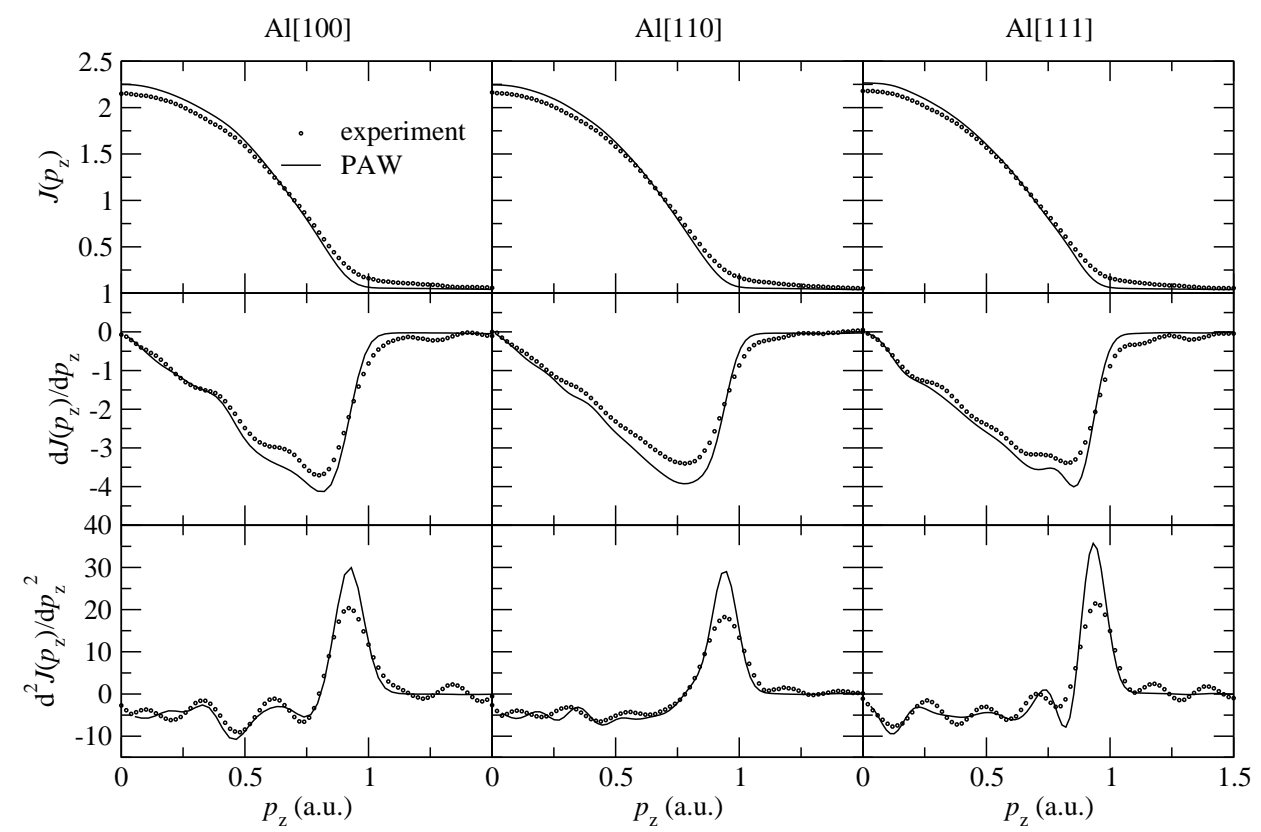

Fig. 3. Valence Compton profiles of $\mathrm{Al}$ along different directions (the first row). Also their first and second derivatives obtained by numerical differentiation are shown (the second and third rows). The theoretical Compton profiles are convoluted with a FWHM of 0.12 a.u. corresponding to the experimental resolution. The experimental valence Compton profiles (from Ref. [51]) have been obtained by subtracting the KKR core profile from the measured ones. 


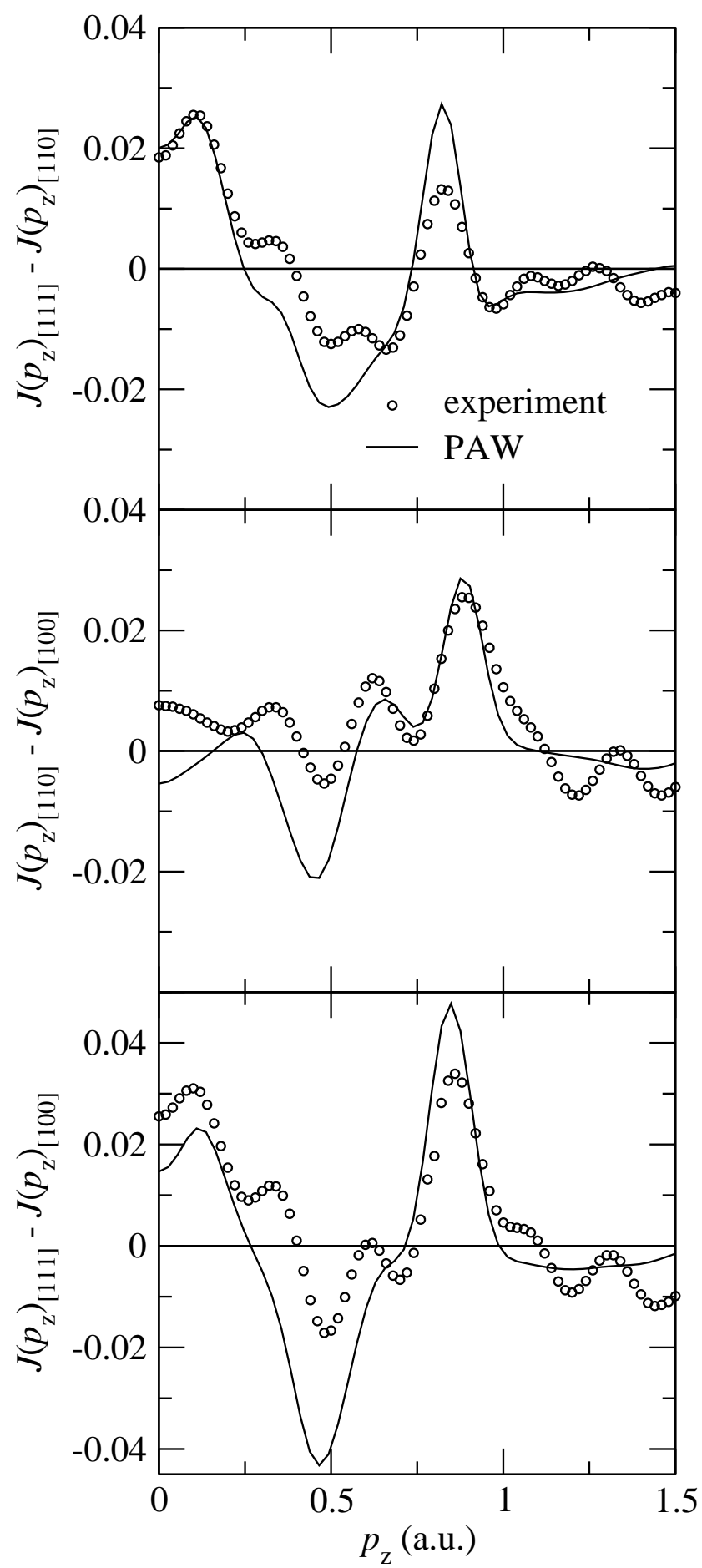

Fig. 4. Bulk Al. Anisotropies between Compton profiles along different directions. The theoretical curves are convoluted with a Gaussian function with a FWHM of 0.12 a.u. corresponding to the experimental resolution. The experimental data is from Ref. [51]. 


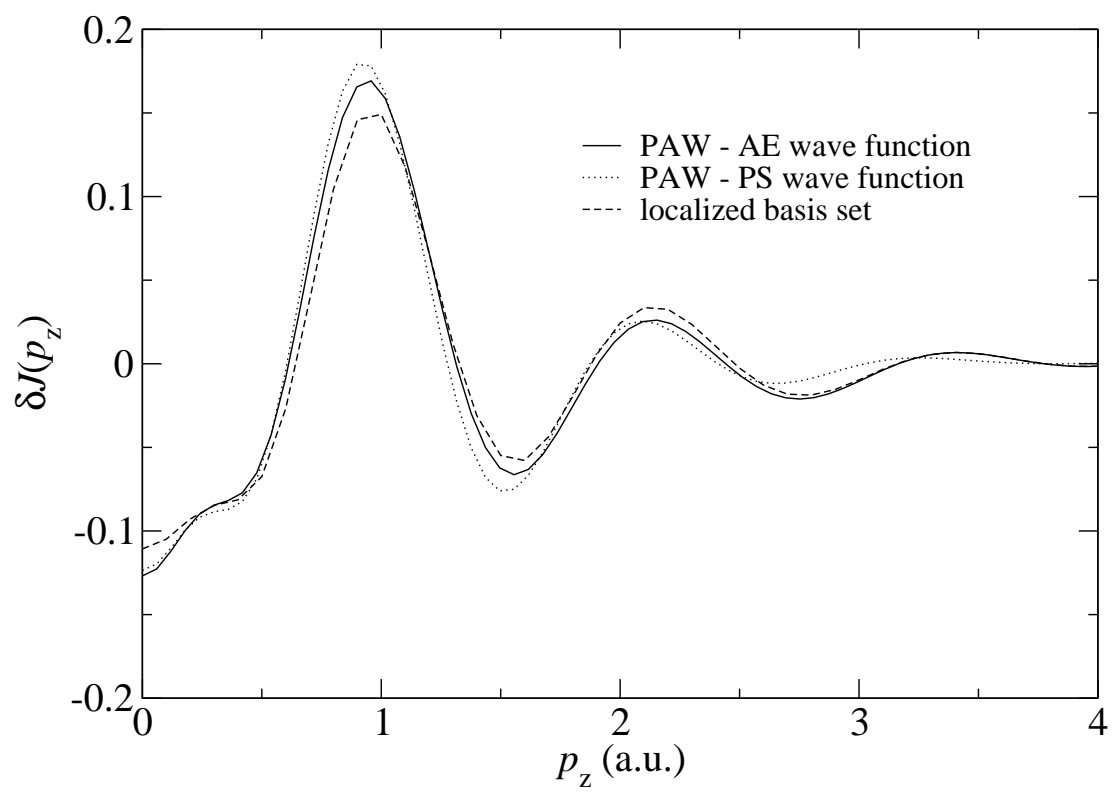

Fig. 5. Compton profile anisotropy for the gas-phase water dimer. 